\title{
Análisis del Comportamiento de la Precipitación Estimada a partir de datosTRMM sobre Áreas de Intensa Deforestación en La Amazonía Legal en el Periodo 2001-2013
}

Isela Leonor Vásquez Panduro Juan Gregorio Rejas Ayuga Otto Correa Rotunno Filho Humberto Barbosa

\section{Resumen}

Con 6,5 millones de $\mathrm{Km}^{2}$, la cuenca Amazónica es el mayor sistema hidrológico del mundo, con descargas estimadas de 209.000 m3s-1, es la mayor extensión continua de bosque tropical. Sin embargo, esta región es el blanco de constantes amenazas, sea por deforestación o por alteraciones climáticas. En este contexto, comprender el funcionamiento del sistema es esencial, sea para auxiliar en la toma de decisiones o estudios de escenarios futuros. Este trabajo tuvo como objetivo analizar la distribución espacio-temporal de la precipitación sobre áreas con alta tasa de deforestación en la cuenca de La Amazonía Legal de Brasil. Para este análisis se utilizaron datos de precipitación mensual estimada por la misión Tropical Rainfall Measuring Mission (TRMM) de NASA, producto 3B43_V6 durante el periodo 2001-2013 y la tasa anual de deforestación estimada por PRODES. Las estimaciones de la precipitación del satélite TRMM muestran buenas correlaciones con los datos de las estaciones observadas, además representa satisfactoriamente las variaciones anuales de precipitación durante el periodo estudiado. Para los años de mayor tasa de deforestación (2001-2006 y 2008) existió un déficit de precipitación representado por las anomalías negativas mientras que en el periodo de recuperación (2009-2013) se obtiene correlaciones positivas. De modo general, se observó que la deforestación parece ejercer ciertas influencias sobre la precipitación. Se propone como línea futura la aplicación extensiva de técnicas de teledetección de los nuevos programas de Observación de la Tierra para el monitoreo de la correlación estudiada en La Amazonía Legal. 
Palabras Clave: Estimación de Precipitación, TRMM, Deforestación, Amazonas.

\section{Abstract}

With 6.5 million km2, the Amazon basin is the largest hydrological system in the world, with estimated discharges of 209,000 m3s-1, it is the largest continuous extension of tropical forest. However, this region is the target of constant threats, whether due to deforestation or climate change. In this context, understanding the functioning of the system is essential, either to assist in decision-making or studies of future scenarios. The aim of this study was to analyze the spatial-temporal distribution of precipitation in areas with high deforestation rates in the Brazilian Amazon basin. For this analysis, NASA's Tropical Rainfall Measuring Mission (TRMM), product 3B43_V6 during the period 2001-2013, estimated monthly rainfall data and the annual rate of deforestation estimated by PRODES are used. Rainfall estimation of the TRMM satellite show good correlations with the data of the observed stations, besides it represents satisfactorily the annual variations of precipitation during the studied period. For the years with the highest rate of deforestation (2001-2006 and 2008), there was a precipitation deficit represented by negative anomalies, while in the recovery period (2009-2013) positive correlations were obtained. Overall, deforestation seems to exert some influence on precipitation. The extensive application of remote sensing techniques using the new Earth Observation programs for monitoring of the correlation studied in The Legal Amazon as a future line are proposed.

Keywords: Rainfall estimation, TRMM, Deforestation, Amazonas.

Isela Leonor Vásquez Panduro, (iselavp@gmail.com), Instituto Alberto Luiz Coimbra de Pós-Graduação e Pesquisa de Engenharia - COPPE. Universidade Federal do Rio de Janeiro - UFRJ, BRAZIL.

Juan Gregorio Rejas Ayuga, (rejasaj@inta.es), Instituto Nacional de Técnica Aeroespacial - INTA, ESPAÑA. Universidad Politécnica de Madrid, ETSI Caminos, Canales y Puertos.

Otto Correa Rotunno Filho, (ottorotunno@oi.com.br), Instituto Alberto Luiz Coimbra de Pós-Graduação e Pesquisa de Engenharia - COPPE. Universidade Federal do Rio de Janeiro - UFRJ, BRAZIL.

Humberto Barbosa, (barbosa33@gmail.com), Universidade Federal do Alagoas - UFAL, BRAZIL, Laboratório de Análise e Processamento de Imagens de Satélites - LAPIS, BRAZIL.

Fecha de recepción: 31 de julio de 2017. Fecha de aprobación: 31 de agosto de 2017. 


\section{INTRODUCCIÓN}

El Amazonas, que representa una de las principales áreas verdes del planeta, viene sufriendo importantes desequilibrios, que pueden provocar desagregación de los suelos y alteraciones del ecosistema natural. Esa desagregación es debida a la deforestación provocada por la expansión de las fronteras agropecuarias, la invasión de las tierras indígenas para la explotación irracional de la madera, la actividad minera y construcción de pistas.

Los bosques amazónicos tienen una serie de ligaciones de retroalimentación con los cambios climáticos que representa una amenaza seria en la existencia de la flora. Un mecanismo es la pérdida de evapotranspiración, que reduce la precipitación a punto que los bosques dejan de ser el tipo de vegetación favorecido por el clima de la región (Shukla et al., 1990). Los bosques serían sustituidos con un tipo de vegetación parecido al que se encuentra en la sabana. Hasta el $60 \%$ de los bosques amazónicos en Brasil podría ser transformado en sabana por el proceso denominado de sabanización (Oyama y Nobre, 2003).

Una de las amenazas estaría relacionada con el aumento de las frecuencias del fenómeno de El Niño. Los eventos de El niño causan sequías en el Amazonas, que a su vez, provee o favorece condiciones para que se produzcan los incendios forestales, como los que ocurrieron en Rondonia en 1997-1998 (Barbosa y Fearnside, 1999). También conduce a pérdidas de carbono del ecosistema de los bosques, incluso en la ausencia de fuego (Tian et al., 1998; Camargo et al., 2004). Los cambios continuos en el equilibrio para los años cuando los bosques pierden carbono (El Niño) y cuando los bosques pueden ganar carbono ("normal" y de la Niña), implican una pérdida a largo plazo de cantidades grandes de carbono.

Teniendo en cuenta que el clima amazónico es caracterizado por un complejo sistema de interacciones de diversos fenómenos meteorológicos que determinan los regímenes de precipitación (Fisch et al., 1998), la topografía y los sistemas de grandes ríos ejercen a su vez un papel importante en los padrones anómalos de la precipitación.

La precipitación en La Amazonía presenta una gran variabilidad espacio-temporal (Figueroa y Nobre, 1990). Debido a la baja densidad de pluviómetros y el difícil acceso en determinados sectores, las estimaciones de precipitación por satélite son una buena alternativa, para contribuir con la mejora del conocimiento de los diferentes regímenes de precipitación, a través de diferentes análisis mucho más detallados sobre la región. 
Además de la cuenca Amazónica, se comportan como un sumidero de humedad para el centro, sudeste y sur de Brasil, así como para el norte de Argentina, incluyendo la cuenca de la Plata, contribuyendo a la ocurrencia de precipitación en esas regiones (Marengo et al., 2004; Marengo, 2006; Satyamurty et al., 2013 ). En este sentido, Satyamurty et al. (2009), mostraron que la actividad convectiva sobre el sur de Brasil y Norte de Argentina, influenciada por el transporte de humedad a través de la frontera sur de la cuenca Amazónica, es realizada por los Jets Bajos Niveis (JBN) al este de los Andes. De acuerdo con Stickler et al., (2013) el vapor de agua disponible para las precipitaciones en Brasil central durante el verano austral viene directamente de La Amazonía. En el mes de diciembre de 2013, un episodio de ZCAS muy intenso y prolongado, alimentado por un flujo de humedad desde La Amazonía con fuerte convergencia en los bajos niveles, generó las inundaciones más severas de las últimas décadas en la cuenca del río Doce (Marengo et al. 2014). Las Iluvias fueron favorecidas por la fuerte convergencia de humedad sobre el Espíritu Santo y la Zona de la Mata Minera, que fue compensada por divergencia de humedad al norte y, especialmente, al sur de la ZCAS, iniciando así el período de sequía sobre São Paulo. Espinoza et al., (2014) observó que la humedad transportada por el JBN de La Amazonia no penetró en el Sudeste de Brasil y fue desviada hacia el oeste de la Amazonia, generando las lluvias intensas e inundaciones en los estados de Rondônia y Acre en el verano de 2014.Vásquez et al., (2017), observaron la relación de la variabilidad y las tendencias en el sudeste de Brasil con los principales sistemas climáticos y oceánicos actuantes durante los periodos lluviosos.

El Amazonas, así mismo, es una región de gran importancia con constantes amenazas por la deforestación o por el cambio climático. En este sentido muchas son las especulaciones sobre las causas de esta sequía en la Amazonía y el centro, sudeste y sur de Brasil, entre ellas variación natural del clima, deforestación de La Amazonía y cambios climáticos globales.

La deforestación, que viene presentando la región del Amazonas Legal es bastante acentuada (con determinadas variaciones por cada año). El trabajo tiene como objetivo analizar la distribución espacio-temporal de la precipitación a través de datos adquiridos del satélite (algoritmo 3B43 de TRMM - Tropical Rainfall Measuring Mission), sobre dos áreas con elevadas tasas de deforestación en el Amazonas Legal, detectadas por el proyecto PRODES (Programa de Cálculo do Desflorestamento da Amazônia) Digita Idel INPE (Instituto Nacional de Pesquisas Espaciais) de Brasil. Se expone así mismo, como línea futura, la aplicación de 
técnicas de teledetección de los nuevos programas de Observación de la Tierra para el monitoreo de la correlación estudiada en La Amazonía Legal.

\section{DATOS Y METODOLOGÍA}

En este trabajo se utilizaron las estigmatizaciones de la precipitación del satélite TRMM, que es equipado con un radar meteorológico, un sensor de microondas pasivo y un sensor óptico en infrarrojo (http://trmm.gsfc.nasa.gov). Específicamente, fueron usados los acumulados medios mensuales desde Enero del 2001 a Diciembre del 2013 del algoritmo 3B43 Versión 6 (3B43_V6),que es una combinación entre los sensores infrarrojos, SSM/I (Special Sensor Microwave/Imager) con informaciones de pluviométricos producidas por GPCC (Global Precipitation Climatology Center). Las salidas de los datos 3B43_V6 muestran el valor de cada 3 horas en cada punto de grado cuya resolución espacial es de $0.25^{\circ} \times 0.25^{\circ}$, donde fue estimada la precipitación mensual. Se puede consultar más detalles en relación con los datos y el algoritmo mencionado anteriormente en http://trmm.gsfc.nasa.gov/3b43.html.

Con relación al área de estudio, se consideraron tres zonas o áreas de test en las que se disponía de un histórico de elevadas tasas de deforestación principalmente en los últimos 10 años, siguiendo las estimativas del Proyecto PRODES y las estadísticas del SAD (Sistema de Alerta de Desmatamento) del IMAZON (Instituto do Homem e Meio Ambiente da Amazônia) (Souza Júnior et al., 2009). Las áreas de test en análisis pertenecen a los estados de Acre, Madeira y Tapajós con resolución de $135 \mathrm{~km}$ x $135 \mathrm{~km}, 135 \mathrm{~km}$ x $110 \mathrm{~km}$ y $82.5 \mathrm{~km}$ x $82.5 \mathrm{~km}$, respectivamente (Figura1).

Para el análisis del comportamiento/evolución de la deforestación sobre las tres áreas elegidas en estudio, se utilizaron los datos del proyecto PRODES digital, objeto del proyecto que consiste en el desarrollo de un método de procesamiento computacional de imágenes, relacionado al mapeo y a las estimativas anuales (a partir del 2000) de deforestación del Amazonas Legal. Esta estimativa es realizada a través del sensor de imágenes TM (Thematic Mapper) del satélite Landsat (cerca de 230 escenas/año) de áreas deforestadas por corte raso y superiores a 6,25 ha, estimando la extensión de la deforestación bruta referente a una data base (Câmaraet al.,2006). Más detalles de PRODES Digital puede ser visto en: www.dpi.inpe.br/prodesdigital/. En este trabajo se han utilizado y analizado los 
datos comprendidos en el período del año 2000 al 2013, adoptando como base y referencia el año 2000. La resolución espacial de los datos es de $90 \mathrm{~m} \times 90 \mathrm{~m}$.

Primeramente, a partir de las estimativas de la precipitación del algoritmo 3B43_V6 ya referido, fueron elaboradas las medias mensuales y anomalías mensuales y anuales de la precipitación a partir de los datos del proyecto PRODES digital. Se ha realizado posteriormente un levantamiento espacial de la deforestación durante el periodo seleccionado y se ha identificado las áreas más deforestadas, analizando así mismo e investigando el comportamiento de la precipitación sobre los tipos de cobertura vegetales presentes en las zonas de estudio.

\section{Deforestación PRODES Digital}

Teniendo en cuenta las tasas de deforestación anual (desde 2000) para toda La Amazonía Legal, disponibles a través del Proyecto PRODES digital, conforme se describe en la metodología, se obtuvo un levantamiento espacial de la deforestación desde el año 2000 al año 2013, como se muestra en la Figura 1. Se observa en general, que el mayor predominio de las áreas con bosque en comparación con las demás clases, viene perdiendo espacio en áreas con presencia de deforestación, es decir, que los efectos de la deforestación evolucionaron de forma intensa durante el periodo en estudio. 

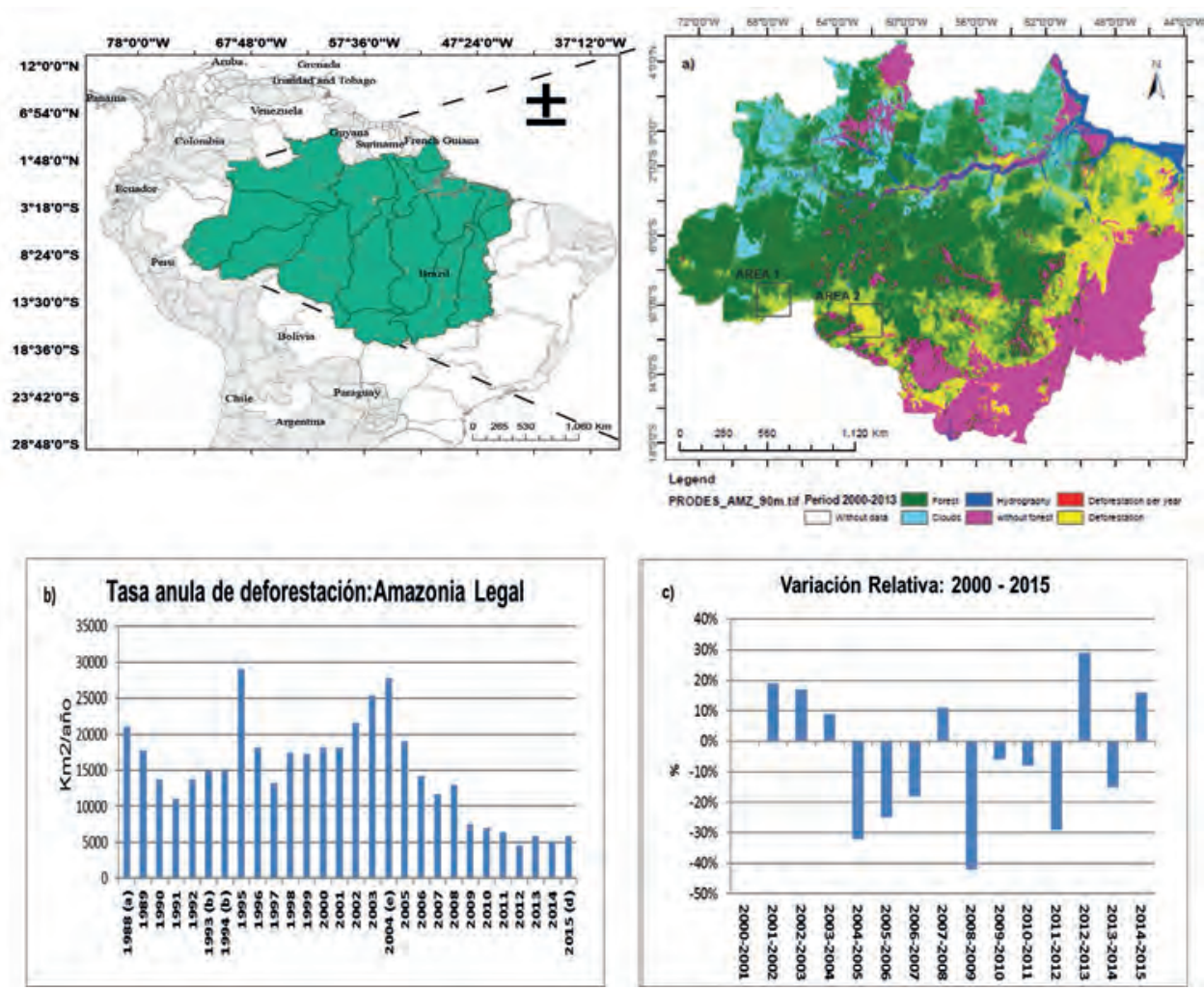

Figura 1. a) Mapa de evolución anual de la deforestación durante el periodo 2000-2013, PRODES digital sobre el Amazonas Legal. b) Evolución anual de la Tasa de deforestación durante el periodo de 1888-2015 (a) Media entre 1977-1988, (b) Media entre 1993-1994, (c) Tasas Anuales Consolidadas, (d) Tasa Estimada, (e) Inició del Plan de prevención y control de la deforestación en el Amazonas (PPCDAM) ). c) Variación relativa de la deforestación periodo 2000-2015.

La transición de la estación seca para la estación lluviosa ocurre entre septiembre y octubre, cuando se observa un aumento significativo de la lluvia en los estados del Amazonas, Acre, Rondônia y Mato Grosso. Durante noviembre, diciembre y enero, los máximos de lluvia varían de $150-350 \mathrm{~mm} / \mathrm{mes}$. Este periodo comprende el verano del hemisferio Sur, cuando hay altas frecuencias de frentes fríos llegando a las regiones tropicales las cuales influencian directamente en la formación de nubes y consecuentemente en la ocurrencia de lluvia en Amazonas. En enero el sistema meteorológico denominado Zona de Convergencia Intertropical (ZCIT) migra y se posiciona al sur del Ecuador, trayendo lluvia para una parte de la región 
oriental del Amazonas. Así en enero aparece un área conteniendo máximos de lluvia en el sur de Amapá, al este de Pará y norte de Maranhão. En los siguientes meses, de febrero a abril, esos máximos aumentan su área espacial y se unen al máximo de lluvia de Mato Grosso y Tocantins, formando una gran área de lluvia sobre el Amazonas Legal. En general, marzo y abril son los meses que se observa los máximos de la estación lluviosa, asociados a la actuación en conjunto con el ZCIT y de frentes fríos posicionados en el Brasil central. En mayo, los máximos comienzan a dislocarse para la región norte de amazona. En junio ocurre la transición de la estación lluviosa hacia la estación seca, con los máximos de lluvia restringidos en la región noroeste del Amazonas y en Roraima.
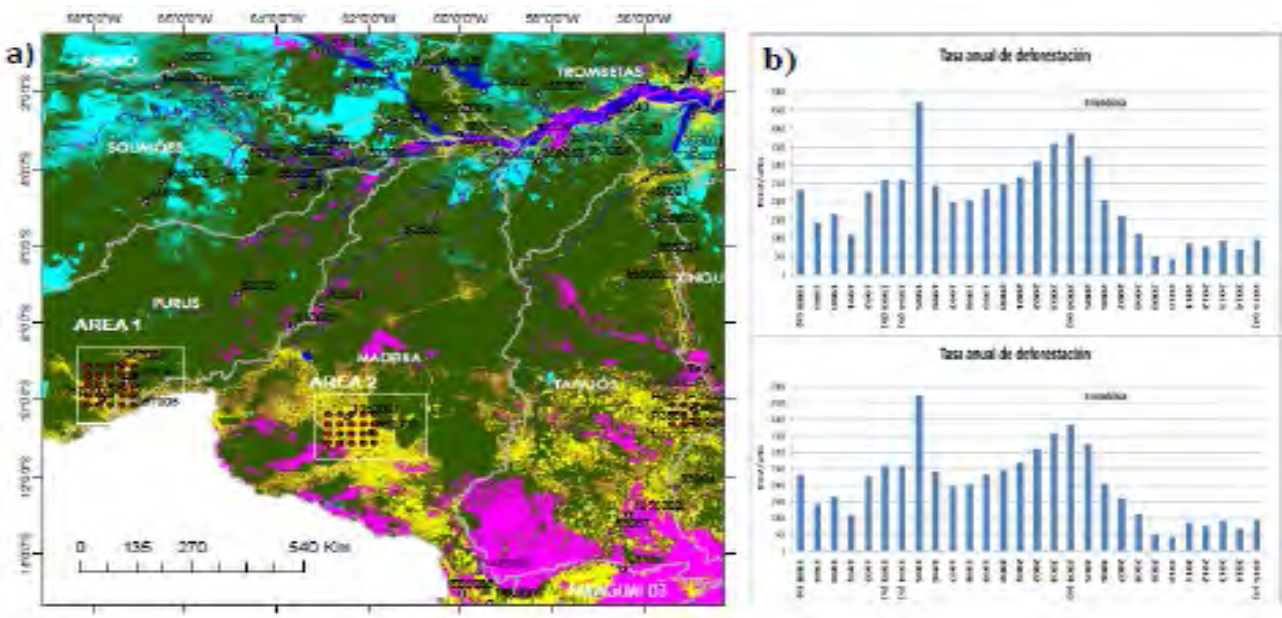

Figura 2. a) Mapa de evolución anual de la deforestación durante el periodo 2000-2013, PRODES digital para el Amazonas Legal sobre áreas de estudio . b) Evolución anual de la Tasa de deforestación durante el periodo de 1888-2015 para los estados Acre -AREA1 y Rondônia-AREA 2. 


\section{RESULTADOS}

\section{Precipitación estimada por el satélite TRMM producto 3B43_V6}

En la Figura 3 se puede observar que las series estimadas estandarizadas de precipitación son bastante aproximadas a las de las estaciones medidas para cada área, acorde con el análisis que permite extraerse a partir de la tabla 1 sobre las correlaciones de las estaciones observadas con las series de las matrices o grillas TRMM estimadas en el conjunto de la región de estudio. Para las series temporales se observa que las anomalías negativas se corresponden principalmente a los años con ocurrencia de eventos de El Niño. También se observa con mayor claridad que en los años que ocurrieron eventos de La Niña las anomalías de la precipitación son significativas. De esta manera se confirma que los años con ocurrencia de $\mathrm{El}$ Niño -Southern Oscillation (ENSO) modulan la precipitación en la región de estudio. Estos resultados están de acuerdo con lo obtenido por otros autores que describen que la variabilidad interanual de la precipitación en el Amazonas estaría asociada a los fenómenos de ENSO (Aceituno, 1988; Ropelewski y Halpert, 1987, 1989; Rao y Hada, 1990; Figueroa y Nobre, 1990; Obregón y Nobre, 1990; Marengo, 1992; Marengo y Hastenrath, 1993; Rao et al., 1996; Poveda y Mesa, 1997; Marengo y Nobre, 2001; Fu et al., 2001; Poveda y Salazar, 2004; Poveda et al., 2006). 

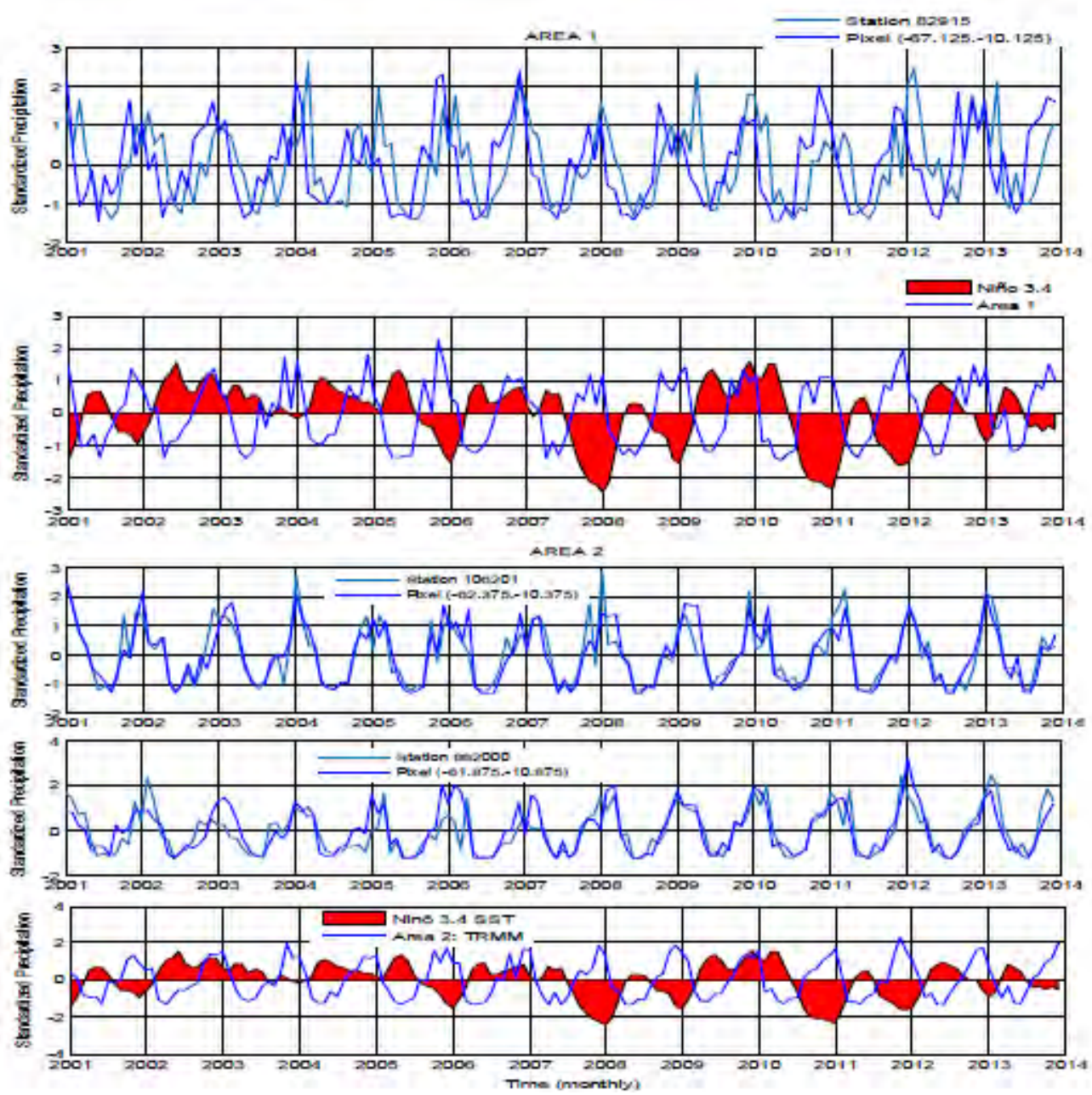

Figura 3. Series temporales de la precipitación normalizada $(\mathrm{mm} / \mathrm{mes})$ observada - TRMM representativa para el AREA, estación 82915 (superior, $1^{\circ}$ y $2^{\circ}$ gráficos) y series temporales de la precipitación normalizada ( $\mathrm{mm} / \mathrm{mes}$ ) observada - TRMM representativa para el AREA 2, estaciones 106201 y 862000 (inferior, gráficos $3^{\circ}, 4^{\circ}$ y $5^{\circ}$ ). 
Tabla1. Correlación al $95 \%$ de significancia entre las estaciones observadas de precipitación y las series estimadas de las grillas TRMM para el conjunto de la región de estudio. Periodo 2001-2013.

\begin{tabular}{|c|c|c|c|c|}
\hline \multicolumn{5}{|c|}{ ESTACAO-TRMM } \\
\hline Station & RR & RMSE & MAE & BIAS \\
\hline 1459003 & 0.53 & 134.27 & 139.67 & 65.95 \\
\hline 359005 & 0.71 & 124.20 & 136.63 & 49.29 \\
\hline 359004 & 0.57 & 120.04 & 136.83 & -3.80 \\
\hline 359001 & 0.48 & 162.98 & 176.55 & 72.26 \\
\hline 463003 & 0.34 & 269.95 & 323.35 & 305.38 \\
\hline 465000 & 0.51 & 169.07 & 191.90 & 54.66 \\
\hline 466002 & 0.58 & 174.10 & 184.26 & 148.53 \\
\hline 655002 & 0.70 & 129.11 & 129.09 & 47.89 \\
\hline 655003 & 0.57 & 143.70 & 154.41 & -2.25 \\
\hline 655004 & 0.44 & 178.76 & 195.69 & 91.70 \\
\hline 455001 & 0.74 & 135.39 & 163.05 & 94.19 \\
\hline 1559006 & 0.51 & 125.96 & 133.70 & 54.90 \\
\hline 63001 & 0.62 & 155.91 & 186.58 & 40.34 \\
\hline 65001 & 0.54 & 114.65 & 139.22 & -4.94 \\
\hline 66000 & 0.45 & 148.65 & 177.42 & 116.61 \\
\hline 260006 & 0.49 & 202.65 & 254.05 & 218.19 \\
\hline 261001 & 0.41 & 185.60 & 215.94 & 28.25 \\
\hline 355001 & 0.77 & 123.15 & 127.11 & 55.97 \\
\hline 356002 & 0.65 & 171.92 & 179.51 & 82.28 \\
\hline 357001 & 0.75 & 129.65 & 148.67 & 37.75 \\
\hline
\end{tabular}




\begin{tabular}{|l|l|l|l|l|}
\hline 357004 & 0.70 & 176.71 & 186.24 & 142.86 \\
\hline 358002 & 0.64 & 148.06 & 161.28 & 32.93 \\
\hline 358003 & 0.70 & 132.96 & 142.30 & 70.71 \\
\hline 363000 & 0.41 & 195.43 & 220.01 & 157.85 \\
\hline 1559000 & 0.51 & 131.67 & 145.41 & 56.31 \\
\hline 1256002 & 0.63 & 138.62 & 153.58 & 8.08 \\
\hline 967004 & 0.63 & 140.69 & 156.45 & 30.26 \\
\hline 967001 & 0.57 & 150.38 & 170.39 & 127.31 \\
\hline 967000 & 0.66 & 115.83 & 138.73 & 28.87 \\
\hline 863005 & 0.64 & 139.57 & 139.90 & 59.95 \\
\hline 763001 & 0.52 & 319.91 & 407.06 & 405.59 \\
\hline 67002 & 0.61 & 135.67 & 152.03 & 14.01 \\
\hline 158003 & 0.51 & 151.71 & 183.54 & 60.45 \\
\hline 162002 & 0.65 & 144.44 & 169.86 & -64.94 \\
\hline 166000 & 0.60 & 155.57 & 183.95 & 129.00 \\
\hline 82113 & 0.47 & 179.40 & 221.13 & 116.69 \\
\hline 82410 & 0.65 & 147.44 & 172.84 & 76.00 \\
\hline 82106 & 0.38 & 187.83 & 230.00 & 42.52 \\
\hline 82326 & 0.50 & 152.24 & 175.09 & 78.54 \\
\hline 82336 & 0.52 & 148.29 & 179.15 & -9.48 \\
\hline 82723 & 0.54 & 135.45 & 159.54 & 70.84 \\
\hline 82317 & 0.65 & 124.49 & 129.32 & -2.29 \\
\hline
\end{tabular}


El área en análisis presentó anomalías relacionadas a los años de los eventos ENSO, siguiendo las figuras 5,6 y 7 . Las anomalías de precipitación registradas que se relacionan con el fenómeno, ocurren en los años de los episodios reconocidos como intensos. Anomalías negativas en los episodios de El Niño de 2002-2003, 2004-2005, 2006-2007 y 2009-2010 y las anomalías positivas en los de La Niña 2001, 2008, 2011 y 2012.
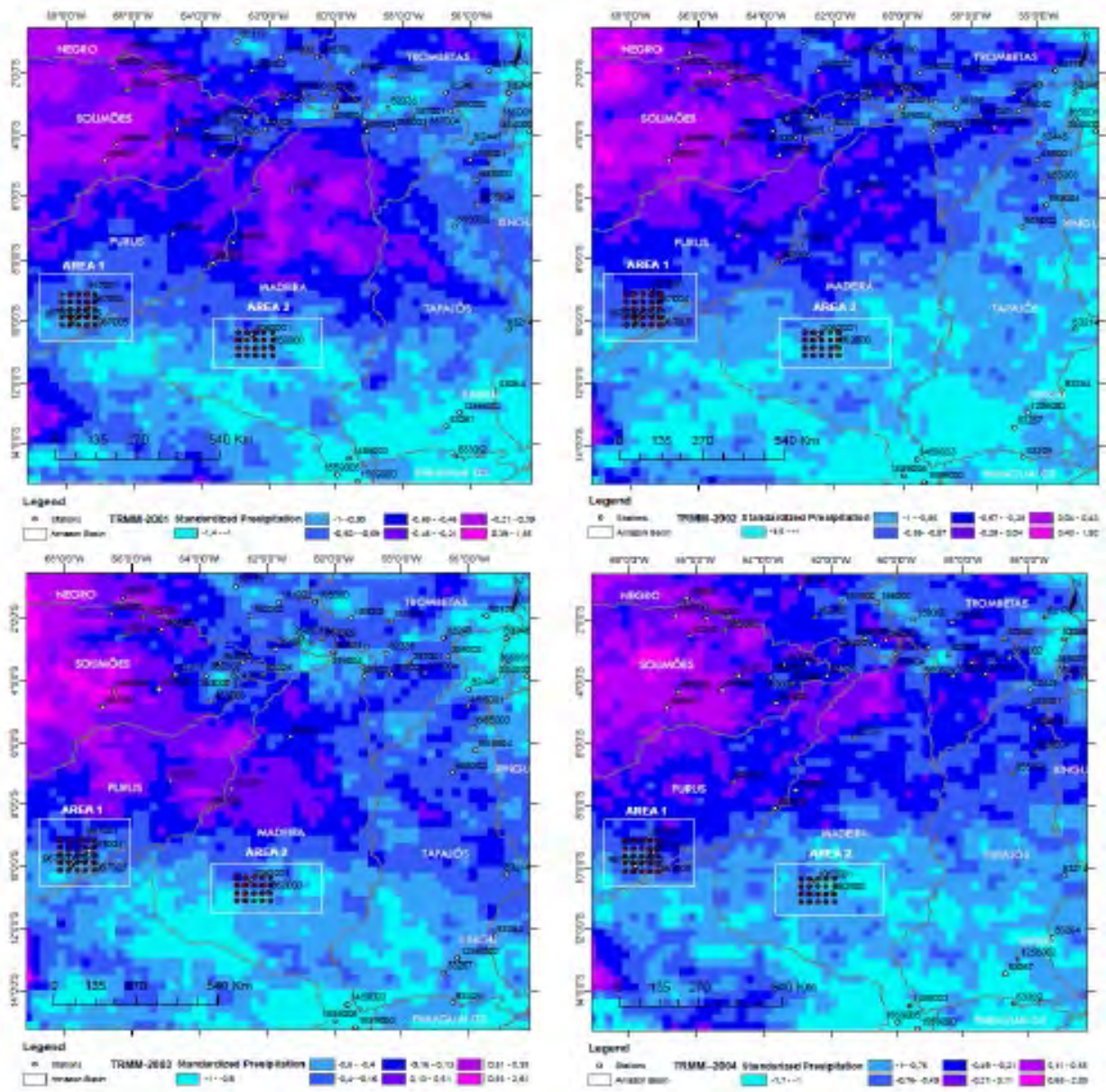

Figura 4. Anomalías totales anuales de la precipitación estimada por el satélite TRMM- 3B43_V6 (enero 2001 diciembre 2004). 
La influencia de El Niño/La Niña (componentes de fenómenos ENSOS) en la climatología de la precipitación en el estado de Amazonas, es observado a través del análisis de la variabilidad espacio-temporal. En el periodo 2001-2008 las distribuciones espaciales de las anomalías negativas están concentradas en las áreas con alta tasa de deforestación. Para el periodo 2009-2013 considerado, en el periodo de recuperación de las áreas deforestadas se observa mayor presencia de anomalías positivas. En este sentido, se observa una posible relación de la deforestación sobre la influencia en el comportamiento de la precipitación coincidiendo con investigaciones en las que se relacionan la reducción de la precipitación sobre la Amazonía con los posibles impactos de antropogénicos como la deforestación y los incendios forestales (Hutyra et al., 2005; Reydon, 2011). Es probable que este hecho haya contribuido a la maximización de los cambios climáticos en la región, ya que los bosques influyen directamente en el clima, causando variaciones de temperatura y humedad relativa (Delire et al., 2011). 


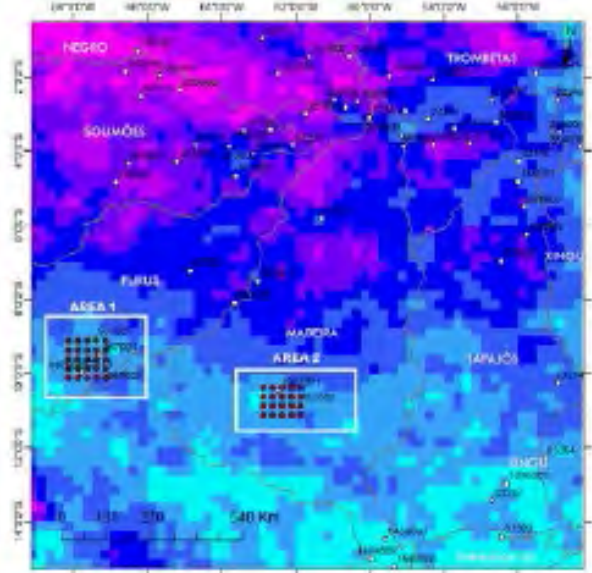

\section{Legend}

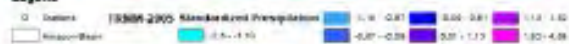

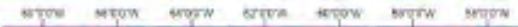

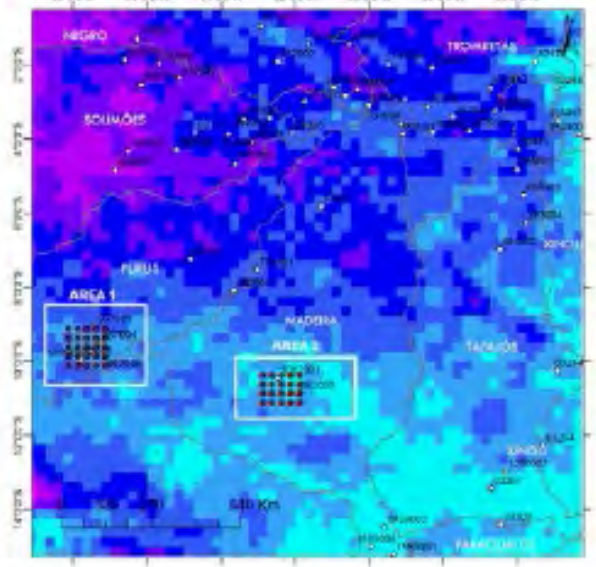

Leaned

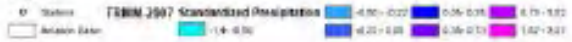

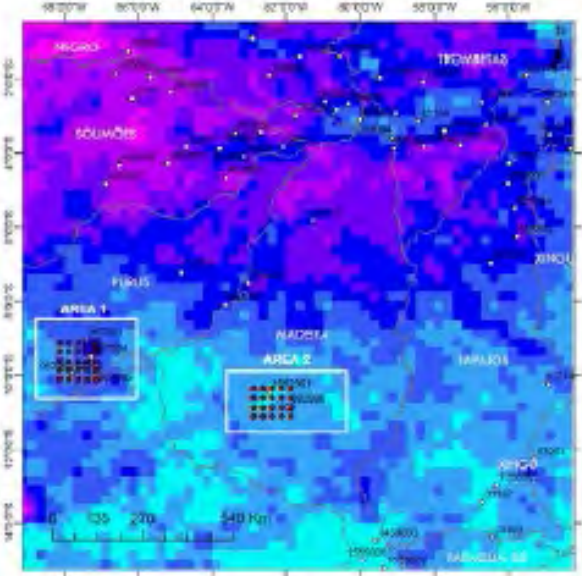

usens

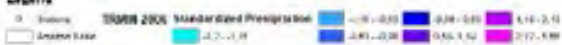

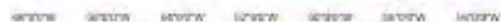

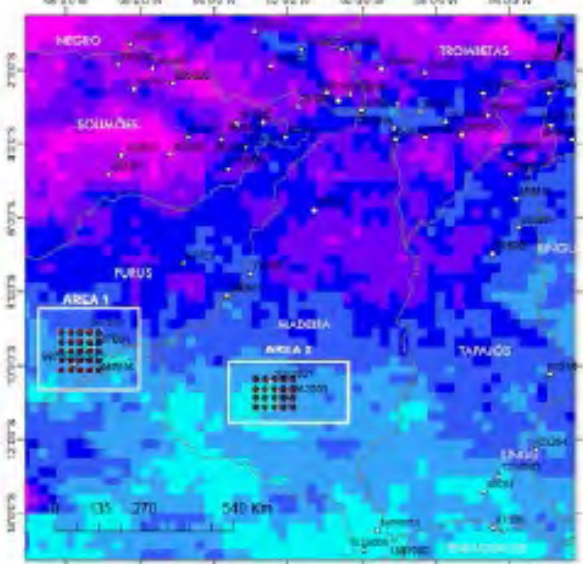

แองละ

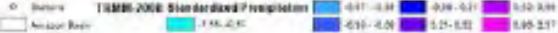

Figura 5. Anomalías totales anuales de la precipitación estimada por el satélite TRMM-3B43_V6 (enero 2005 diciembre 2008). 

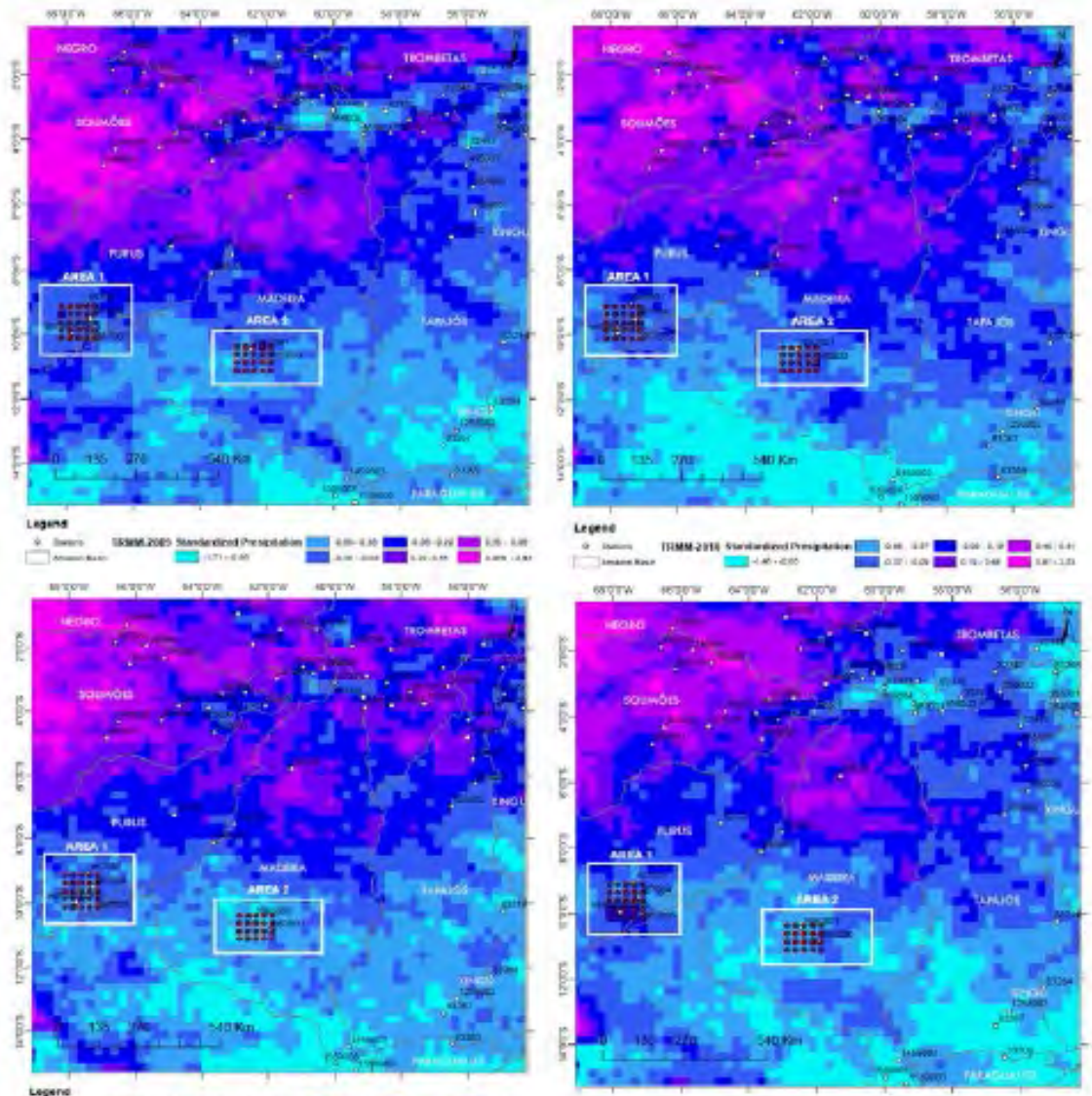

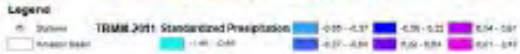
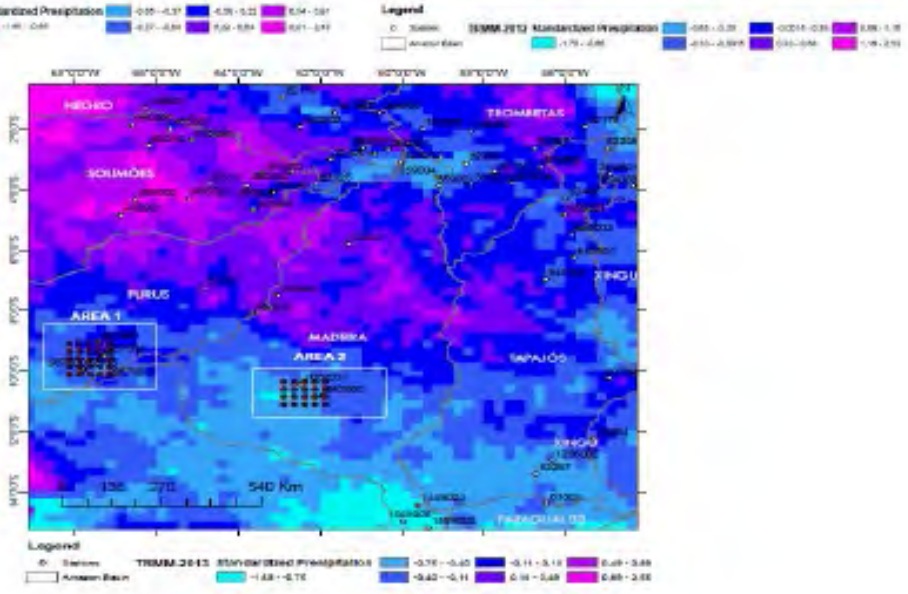

Figura 6. Anomalías totales anuales de la precipitación estimada por el satélite TRMM- 3B43_V6 (enero 209 diciembre 2013). 


\section{LÍNEA FUTURA DE INVESTIGACIÓN}

Una vez validados los diferentes modelos, uno de los objetivos para los próximos años será desarrollarlos en base a un ajuste e intercalibración sobre las áreas de test a partir de medidas in situ y análisis propios de los diferentes programas espaciales globales disponibles, principalmente Sentinel 2 de la Agencia Espacial Europea (ESA, en sus siglas en inglés) y Landsat-8 de NASA. El objetivo será probar los modelos ya validados en estos nuevos datos y ajustarlos a zonas concretas de test que permitan un análisis comparativo de intercalibración, tanto en Suramérica y Centroamérica como en Europa (Rejas et al., 2007, 2013; Algeet et al., 2013; Essono et al., 2015; Marchamalo et al., 2014). Se ha realizado en este sentido una primera aproximación (Vásquez et al., 2016) para estimar tiempos de computación y realizar análisis exploratorios de los datos referidos sobre las áreas de test (Figura 7).

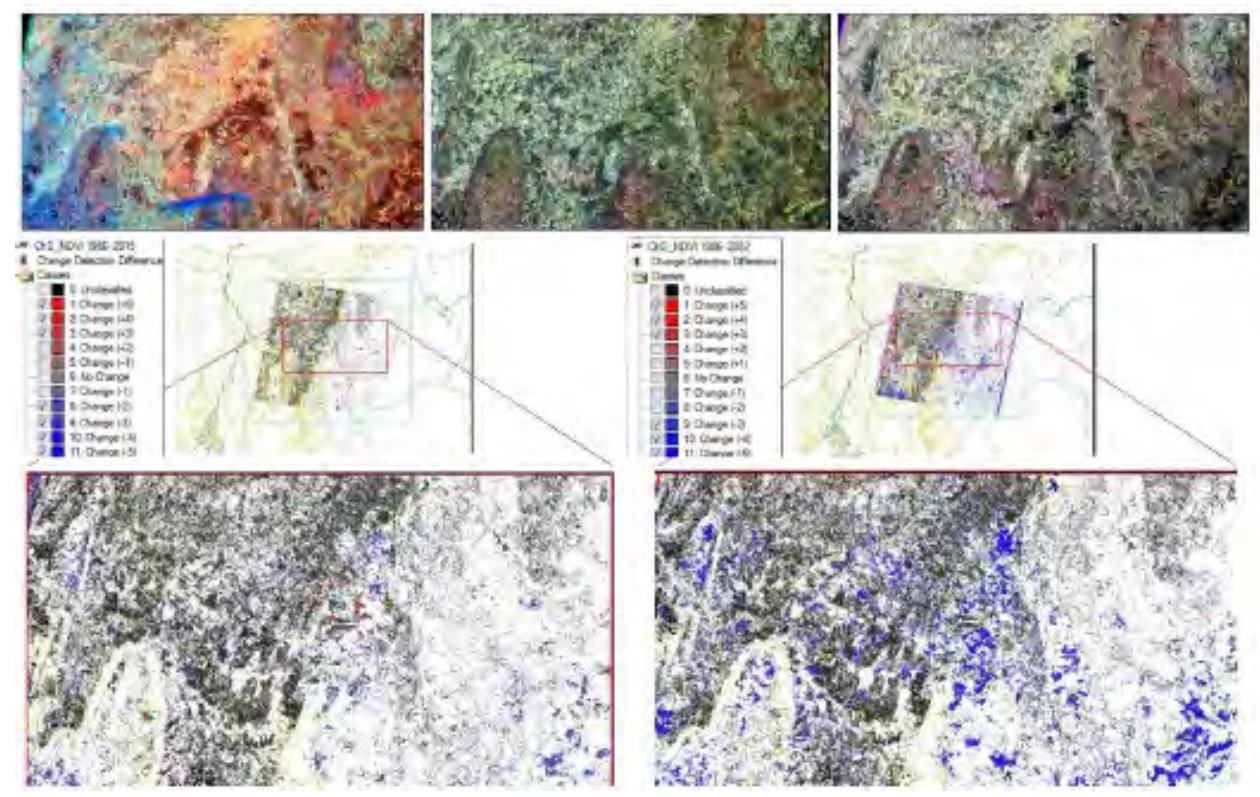

Figura 7. Ejemplo de sub-escenas de OLI (L (), ETM + (L7) y TM (L5) para 2015, 2002 y 1986, respectivamente (arriba) y ejemplo de detección de cambios NDVI en la cuenca del río Sâo Franciso entre 1986 y 2015 (inferior izquierda) y 1986 y 2002 (inferior derecha). Su cuenca representativa Pedro do Rio $\left(411 \mathrm{~km}^{2}\right)$ está ubicada en la región montañosa del Estado de Río de Janeiro, Brasil, donde el trimestre más húmedo se corresponde con diciembre-enero-febrero constituyendo el $45 \%$ del total de la precipitación anual. 
En esta aproximación se han aplicado técnicas exploratorias de imagen en series de los sensores Sentinel 2 (S2), OLI, ETM+ y TM, cuyo objetivo es contrastar parámetros biofísicos y obtener un primer estado de la vegetación y los suelos en el entorno al área estudiada. Se ha estimado y minimizado el error de las imágenes primeramente, realizando un chequeo de las transformaciones radiométricas y geométricas contenidas en el nivel de proceso L1 de las imágenes a partir de datos medidos en campañas de supervisión.

En este primer ensayo se ha buscado evaluar la influencia de la cubierta de vegetación, abundante en la zona de estudio, en el análisis espectral de imágenes S2, OLI, ETM+ y TM. En este sentido, se han calculado índices de vegetación y de suelo para el conjunto de las imágenes de prueba. Se ha generado el NDVI 0 Normalized Difference Vegetation Index (Rouse et al., 1974) para evaluar el área foliar según la conocida expresión:

$$
N D V I=\frac{\rho_{\mu N I R}-\rho_{\mu R}}{\rho_{\mu N I R}-\rho_{\mu R}}
$$

donde $\rho \mu N I R$ es la reflectancia de banda del infrarrojo próximo y $\rho \mu R e s$ la reflectancia de banda del rojo. Para estimar la reflectancia transformada por la absorción de la clorofila en relación con la vegetación del suelo (Berni et al., 2010) se ha generado el ratio entre el índice TCARI (Transformed Chlorophyll Absorption Ratio Index) y OSAVI (Optimized Soil Adjusted Vegetation Index) particularizado para los sensores bajo test según la expresión:

$$
\begin{aligned}
& \text { TCARI OSAVI }=3 \cdot\left[\left(\rho_{\mu 700}-\rho_{\mu 670}\right)-0.2 \cdot\left(\rho_{\mu 700}-\rho_{\mu 550}\right) \cdot\right] \\
&\left(\rho_{\mu 700}-\rho_{\mu 670}\right)(1+0.16) \cdot\left(\rho_{\mu 800}-\rho_{\mu 670}\right) \\
&\left(\rho_{\mu 800}-\rho_{\mu 670}+0.16\right)
\end{aligned}
$$

El objetivo a medio plazo es comprender las relaciones temporales del estado de la vegetación y de los suelos que podemos extraer a partir de los datos registrados por los Programas de Observación de la Tierra, con la evolución de la precipitación en general, y con las anomalías mensuales y anuales de la precipitación en 
particular, y sus efectos a su vez en la evolución de la deforestación y en procesos de desertificación en zonas semiáridas.

\section{CONCLUSIONES}

A través de los datos estimados de precipitación de la misión TRMM (3B43_V6), de las estimaciones anuales de la deforestación del Proyecto PRODES Digital, se observa en general que la deforestación parece ejercer una determinada influencia en el comportamiento de la precipitación en las zonas de La Amazonía Legal de Brasil estudiadas en el período 2001-2013.

Los episodios de La Niña y El Niño provocan impactos negativos de inundaciones y sequías motivados por la variabilidad climática interanual, en torno de las medias climatológicas, lo que se ha podido observar en los resultados analizados para las zonas de test estudiadas.

La Amazonía sufre deforestación no solo por eventos ENSO, mas también por los cambios en el uso del suelo que puede interferir significativamente en el clima en la región.

Con esto, el análisis del comportamiento y la detección de las áreas deforestadas obtenidos en el presente artículo gracias al Proyecto PRODES, se presentó como una herramienta de suma importancia para estudios de impacto, en este caso particular por la precipitación, en el ciclo hídrico debido a la deforestación, Por tanto, basados en estos resultados, el presente trabajo ha permitido contribuirá una mejor compresión del impacto causado por la deforestación en la precipitación sobre La Amazonía Legal.

Finalmente, en base a unos primeros ensayos y análisis exploratorios a partir de Sentinel 2 y OLI de Landsat 8 , se observa el potencial para la implementación de la metodología expuesta en procesos futuros de análisis e intercalibración multiescenario.

\section{AGRADECIMIENTOS}

Inicialmente, los autores desean reconocer el apoyo brindado por el Consejo Nacional de Desarrollo Científico y Tecnológico (CNPq) con la beca otorgada al primer autor. Los autores agradecen al Proyecto PRODES por el acceso a los datos 
y algoritmos utilizados en el presente artículo, así como al programa TRMM de NASA. Los autores desean también dar las gracias al Programa de Ingeniería Civil del Instituto Alberto Luiz Coimbra de Pós-Graduación e Investigación de Ingeniería (COPPE) - Universidad Federal de Río de Janeiro (UFRJ), con el apoyo del Laboratorio de Recursos Hídricos y Estudios Ambientales LABH2O). Los autores quisieran también reconocer el apoyo de la CAPES, a través de la CAPES Edital MEC 03/2015 - BRICS, Consejo Nacional de Desarrollo Científico y Tecnológico (CNPq), a través del CNPq Edital Universal No. 14/2013 - proceso 485136 / 2013-9 y CNPq Edital No. 12/2016 - proceso 306944 / 2016-2, Fundación de Amparo a la Investigación del Estado de Río de Janeiro (FAPERJ) mediante el apoyo a través del Proyecto FAPERJ - Pensa Rio - Edital 34/2014 (2014-2018) - E-26 / 010.002980 I 2014, que se centran en la lluvia-escorrentía y el modelado meteorológico en conjunto con el uso de imágenes asesoramiento remoto.

\section{BIBLIOGRAFÍA}

- Aceituno, P.F. (1988). On the functioning of the Southern Oscillation in the South American Sector. Part I: Surface climate .Mon. Wea. Rev., 116, 505-524.

- Algeet, N., Fernández, J., Lianes, E., Marchamalo, M., Martínez, R. and Rejas, J.G. (2012). Manejo Integral de Agua y Suelo en Centroamérica. Bases Científicas para el Desarrollo Rural Comunitario. Programa de Cooperación Comunidad Agua y Bosque en Centroamérica, Ed. UPM Madrid (España) ISBN: 84-7493-467-2.

- Barbosa, R. and Fearnside, P.M. (1999). Incêndios na Amazônia brasileira: estimativa da emissão de gases do efeito estufa pela queima de diferentes ecossistemas de Roraima na passagem do evento "El Niño" (1997/98). Acta Amazonica 29: 513-534.

- Câmara, G., Valeriano, D. M., e Soares, J. V. (2006). Metodologia para o Cálculo da Taxa Anual de Desmatamento na Amazônia Legal. São José dos Campos. INPE, 2006. 24 p. Disponible en<http://www.obt.inpe.br/prodes>.

- Camargo M.S., Shimizu L.K., Saito M.A., Kameoka C.H., Mello S.C. and Carmello Q.A.C. (2004). Crescimento e absorção de nutrientes pelo lisianthus (Eustoma grandiflorum) cultivado em solo. Horticultura Brasileira 22: 143- 146. 
- Delire, C.; Noblet-Ducoudré, N.; Sima, A.; Gouirand, I.( 2011). Vegetation Dynamics Enhancing Long-Term Climate Variability Confirmed by Two Models. Journal of Climate, Boston, v.24, n. 9, p. 2238-2257.

- Esono, S., Rejas, J.G., Bonatti, J., Martínez, R., Marchamalo, Mi., Algeet, N. y Fernández, J. (2015). Análisis de patrones espectrales del terreno y cambios hidrológicos a partir de datos multisensor en espacios naturales del sur de Costa Rica. CIENCIAS ESPACIALES (ISSN:2225-5249), V8, № 2 ， 261277.

- Espinoza, J. C. et al.( 2014). The extreme 2014 flood in South-Western Amazon basin: The role of Tropical-Subtropical South Atlantic SST gradient. Environmental Research Letters, v. 9, n. 12, p. 124007.

- Figueroa, N. and Nobre, C.A. (1990). Precipitation distribution over Central and Western Tropical South America, Climanálise, 5, 36-48.

- Fisch, G., Marengo, J. A. and Nobre, C. A. (1998). Uma revisão geral sobre 0 clima da Amazônia. Acta Amazonica, 28(2), 101-126.

- Fu, R., Chen, M., Li, W. and R. E. Dickinson (2001). How do tropical sea surface temperatures influence the seasonal distribution of precipitation in the equatorial Amazon?Int.J. Climatology, 14, 4003- 4026.

- Hutyra L.R, Munger J.W, Nobre C.A, Saleska S.R, Vieira S.A, Wofsy S.C.(2005). Climatic variability and vegetation vulnerability in Amazonia. Geophys. Res. Lett. 32, 4 doi:10.1029/2005GL024981.

- Marchamalo, M., Algeet, N., Fernández, J., Rejas, J.G., Martínez-Frías, J., Martínez, R. and Bonatti, J. (2014). Potential Applications of Sentinel-2 In Land Cover Analysis In The Tropics: Lessons Learned in Costa Rica. Sentinel-2 for Science Workshop, Frascati (Italy) ，20th, 21st and 22nd May 2014.

- Marengo, J. A. (1992). Interannual variability of surface climate in the Amazon Basin, Int. J. Climatology, 12, 853-863.

- Marengo, J. A. (1995). Interannual variability of deep convection over the tropical South America sector as deduced from ISCCP C2 data, Int. J. Climatology, 15, 995-101.

- Marengo, J. A. and S. Hastenrath (1993). Case studies of extreme climatic events in the Amazon basin.J. Climatology 6, 617-627. 
- Marengo, J. A. and Nobre, C. (2001). General Characteristics and variability of Climate in the Amazon Basin and its Links to the Global Climate System, The hydroclimatological framework of Amazonia, Biogeochemistry of Amazonia, edited by J. Richey, M. MacClaine, and R. Victoria, pp. 17-41, Cambridge University Press.

- Marengo, J. A.( 2006). On the hydrological cycle of the amazon Basin: A historical review and current state-of-the-art. Revista Brasileira de Meteorologia 21(3): 1-19.

- Marengo, J. A., Fisch, G., Morales, C. et al. (2004).Diurnal variability of rainfall in southwest amazonia during the LBA-TRMM field campaign of the austral summer of 1999. Acta Amazonica. 34, 593-603.

- Marengo, J. A.; Alves, L. M. (2016). Crise hídrica em São Paulo em 2014: seca e desmatamento. Geousp - Espaço e Tempo (Online), v. 19, n. 3, p. 485-494, mês. ISSN 2179-0892.

- Obregón, G. and Nobre, C.A. (1990). Principal Component Analysis applied to the rainfall in Amazonia.Climanálise, 5, 35-46.

- Oyama, M. D. and C. A. Nobre (2003). A new climate-vegetation equilibrium state for Tropical South America.Geophysics Res. Lett., 30, 2199, doi:10.1029/2003GL018600, 23.

- Poveda G. and Mesa, O.J. (1997).Feedbacks between hydrological processes in tropical South America and large scale oceanic atmospheric phenomena.J. Climatology, 10, 2690-2702.

- Poveda, G. and Salazar, L.F. (2004). Annual and interannual (ENSO) variability of spatial scaling properties of a vegetation index (NDVI) in Amazonia, Remote Sensing and Environment, 93, 391-401.

- Poveda, G., Waylen, P.R. and Pulwarty, R. (2006). Modern climate variability in northern South America and southern Mesoamerica, Palaleoclimatology, 234, 3-27.

- Rao, V. B. and K. Hada (1990). Characteristics of rainfall over Brazil: annual variations and connections with the southern oscillation. Theoretical and Applied Climatology, 42, 81-91. 
- Rao, V. B., Cavalcanti, I.F. and K. Hada (1996). Annual variations of rainfall over Brazil and water vapor characteristics over South America, J. Geophys. Res., 101, D21, 26539-26551.

- Reydon, B. P.(2011). O desmatamento da floresta amazônica: causas e soluções. Política Ambiental, Belo Horizonte, v. 1, n. 8, p. 143-155.

- Rejas, J.G., Martínez, R. and Malpica J. A. (2007). Hyperspectral remote sensing application for semi-urban areas monitoring. IEEE, ISBN-1-4244-07117 catalog number 07EX1577.

- Rejas, J.G., Martínez, R., Marchamalo, M., Bonatti, J. and Martínez-Frías, J. (2013). Spectral Signature Analisys for Anomaly Detection in the Central Volcanic Range, Costa Rica. Implications in Planetary Geology. Mathematics of Planet Earth ISSN 2193-8571.

- $\quad$ Rouse, J.W., Haas, R.H., Schell, J.A., Deering, D.W. and Harlan, J.C.(1974). Monitoring the vernal advancements and retrogradation of natural vegetation. NASA/GSFC, Greenbelt, D, p. 371, 1974. Final Report.

- Ropelewski, C. F. and M. S. Halpert (1987). Global and regional scale precipitation patterns associated with the El Niño/Southern Oscillation, Mon. Wea. Rev., 115, 1606-1626.

- Ropelewski, C. F. and M. S. Halpert (1989). Precipitation patterns associated with the high index phase of the Southern Oscillation, J. Clim., 2, 268-282.

- Satyamurty, P., Castro, A.A., Tota, J., Gularte, L. E. S., Manzi, A.O. (2009). Rainfall trends in the Brazilian Amazon Basin in the past eight decades. Theoretical and Applied Climatology, DOI10.1007/s00704-009-0133-x.

- Satyamurty, P.; Costa, C. P. W.; Manzi, A. O.( 2013).Moisture source for the amazon basin: a study of contrasting years. Theoretical and Applied Climatology, v. 111 , n. 1-2, p. 195-209. 1, 10, 13, 14, 22, 27, 36, 49, 61

- Shukla, J., Nobre, C. and P. Sellers, 1990. Amazon deforestation and climate change. Science 247: 1322-25.

- Souza, C., Cochrane, M., Sales, M., Monteiro, A. and Mollicone, D. (2009). Integrating forest transects and remote sensing data to quantify carbon loss due 
to forest degradation in the Brazilian Amazon. Forest Resource Assessment Working Paper, 161, 1-17.

- Stickler, C. M.; Coe, M. T.; Costa, M. H.; Nepstad, D. C.; Macgrath, D. G.; Dias, L. C. P.; Rodrigues, H. O.; Soares-Filho, B. S.(2013). Dependence of hydropower energy generation on forests in the Amazon Basil at local and regional scale. PNAS, n. PNAS Early Edition, p. 2-6.

- Tian, H., Mellilo, J. M., Kicklighter, D. W., McGuire, A. D., Helfrich III, J.V.K., Moore III, B. and Vörösmarty, C., (1998). Effect of interannual climate variability on carbon storage in Amazonian ecosystems. Nature 396: 664-667.

- Vásquez, I.L., Rejas, J.G. and Rotunno, O.C. (2016). The Hydrological Cycle of the Amazon Basin: Climatic Impact of Deforestation. NASA Direct Readout Conference NDRC-9, Valladolid (Spain).

- Vásquez P., I.L., de Araujo, L.M.N., Molion, L.C.B. et al. (2017). Historical analysis of interannual rainfall variability and trends in southeastern Brazil based on observational and remotely sensed data. Climate Dynamics, doi. org/10.1007/s00382-017-3693-y 